\title{
Recent advances of GOLM1 in hepatocellular carcinoma
}

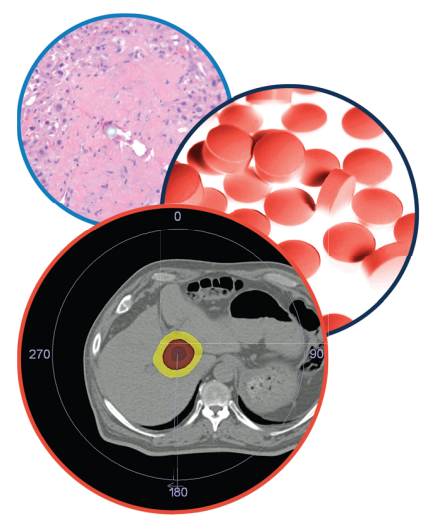

Jiuliang Yan ${ }^{\ddagger 1,2}$, Binghai Zhou ${ }^{\ddagger 1,2}$, Hui Li ${ }^{1,2}$, Lei Guo ${ }^{1,2}$ \& Qinghai Ye*,1,2

${ }^{1}$ Department of Liver Surgery \& Transplantation, Liver Cancer Institute \& Zhongshan Hospital, Fudan University, Shanghai, 200032, China

${ }^{2}$ Key Laboratory of Carcinogenesis \& Cancer Invasion (Fudan University), Ministry of Education, Fudan University,

Shanghai, 200032, China

*Author for correspondence: ye.qinghai@zs-hospital.sh.cn

${ }^{\ddagger}$ Authors contributed equally

Hepatocellular carcinoma (HCC) is one of the most common liver malignancies and is a leading cause of cancer-related deaths. Most HCC patients are diagnosed at an advanced stage and current treatments show poor therapeutic efficacy. It is particularly urgent to explore early diagnosis methods and effective treatments of HCC. There are a growing number of studies that show GOLM1 is one of the most promising markers for early diagnosis and prognosis of HCC. It is also involved in immune regulation, activation and degradation of intracellular signaling factors and promotion of epithelial-mesenchymal transition. GOLM1 can promote HCC progression and metastasis. The understanding of the GOLM1 regulation mechanism may provide new ideas for the diagnosis, monitoring and treatment of HCC.

First draft submitted: 23 February 2020; Accepted for publication: 21 May 2020; Published online: 29 June 2020

Keywords: diagnosis $\bullet$ GOLM1 $\bullet$ hepatocellular carcinoma $\bullet$ immune system $\bullet$ prognosis

Hepatocellular carcinoma (HCC) is the third most common cause of cancer-related deaths worldwide [1]. The poor prognosis is due to extensive regional invasion and distant metastases at the time of diagnosis. Therefore, it is necessary to explore effective early diagnosis and identify reliable biological and clinical markers. The relationship between Golgi apparatus and tumors is receiving growing attention [2,3]. New Golgi proteins and Golgi-related proteins have been discovered. Among them, GOLM1 (also known as GP73 or GOLPH2) is the most anticipated tumor biomarker. A growing number of studies have shown that the abnormal expression of GOLM1 is associated with HCC and may become a new target for the prevention, monitoring and treatment of HCC [4-7]. In this review, we discuss the recent advances of GOLM1 in HCC and present an overall view of future research.

\section{Overview of GOLM1 protein}

Structure of GOLM1

Kladney et al. first reported the isolation and characterization of GOLM1 in the year 2000 [8]. GOLM1 is a Golgi membrane protein mainly expressed in epithelial cells and upregulated in response to viral infection, its coding gene is located on human chromosome 9q21.33 with a full-length sequence of $3042 \mathrm{bp}$. It contains a $1200 \mathrm{bp}$ open reading frame including two in-frame coding regions to produce 400 or 391 amino acids respectively. GOLM1 contains a typical Type II transmembrane protein structure, with a short $\mathrm{N}$-terminal transmembrane region and a long complex $\mathrm{C}$-terminal domain. It is highly hydrophilic, while the $\mathrm{N}$-terminus is hydrophobic. There is a potential signal peptidase cleavage site between amino acids 28 and 29; in addition, the $\mathrm{N}$-terminus also contains an N-myristoylation consensus sequence (GLGNGRRS). This structure may be related to signal transduction and protein phosphorylation/dephosphorization reactions. Highly acidic regions with unknown function and five potential glycosylation sites are present in the C-terminus. Several coiled-coil domains at the C-terminus are adjacent to the transmembrane region (Figure 1) [9]. These coiled coils are indispensable in the processes of binding, docking and exchange with vesicles containing various proteins, lipids and nucleic acids $[8,10,11]$.

Future Medicine 


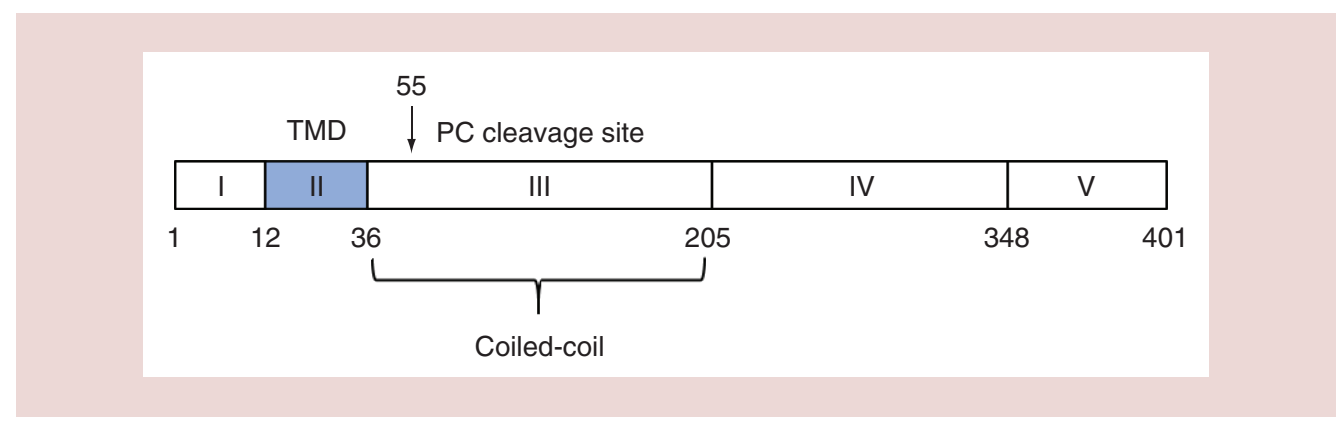

Figure 1. Schematic structure of GOLM1. Based on sequence and structure analyses, region I (cytoplasmic domain), region II (TMD) and region III-V (Golgi lumen domains) are indicated. The amino acid numbers of the positions are indicated.

TMD: Transmembrane domain.

\section{Expression distribution \& subcellular localization of GOLM1}

GOLM1 can be expressed in a variety of human tissues, predominantly in epithelial cells. For example, GOLM1 is mainly expressed in the columnar epithelial cells of the intestinal gland in the colon; in the ciliated columnar epithelial cells of the bronchioles in the lung; in the glandular epithelial cells in the prostate. In normal liver tissue, GOLM1 is mainly found in the epithelial cells of bile duct, with either absent or weak expression in hepatocytes. However, hepatitis B virus and adenovirus infection significantly increases GOLM1 expression in hepatocytes, but does not change GOLM1 level in biliary cells $[8,12,13]$.

GOLM1 exhibits the perinuclear localization in cells and colocalizes with Golgi membrane protein giantin [8]. One study from Puri et al. showed that GOLM1 colocalized with the cis-Golgi protein GPP130 and did not overlap with the localization of the trans-Golgi network protein TGN46, suggesting that the GOLM1 protein localized to the cis-Golgi [14]. This study also demonstrated that GOLM1 is synthesized in the endoplasmic reticulum and the major fraction is then transported to the cis-Golgi, while a small proportion of GOLM1 proteins are transported to the cell surface and then GOLM1 on the cell surface is endocytosed and returned to the Golgi through the endosomal pathway. GOLM1 is also a kind of secreted protein and is secreted form can be detected in both blood and urine. Bachert et al. found that the proprotein convertase site $\mathrm{R}^{52} \mathrm{VRR}^{55}$ was present in the GOLM1 protein sequence (Figure 1) [15]. During the Golgi-to-endosome transport process, GOLM1 is cleaved by the furin precursor protease to form a secreted protein separating from the transmembrane region.

\section{Function of GOLM1}

The biological function of GOLM1 in cells and tissues is not well understood. GOLM1 is expressed in most human tissues. Therefore, it can be speculated that GOLM1 may belong to the housekeeping genes. The significant variations of GOLM1 mRNA and protein levels in different tissues indicate that it may have tissue-specific regulatory functions. It is reported that GOLM1 can maintain the structural integrity of the Golgi complex under cellular stress [12]. Wright et al. used genetic defects methods to establish a mouse model lacking the C-terminus of GOLM1 and found that the overall survival rate was lower than that of wild-type mice, especially in female mice [16]. In addition, these mice were prone to having various degrees of damage to the kidney and the liver, such as focal segmental glomerulosclerosis and hepatic steatosis. These findings suggest that GOLM1 may play an important role in maintaining normal cell function. GOLM1 is a Golgi resident protein that is not normally secreted by cells. However, under some disease conditions, GOLM1 can be released into the cytoplasm by endoproteolytic cleavage at the proprotein convertase site and transported through the endosomes to the cell surface [15].

\section{Significance of GOLM1 in the diagnosis of HCC}

GOLM1 protein level in serum is very small or even absent in healthy individuals compared with the significantly increased level in primary hepatic carcinoma patients [17], indicating that GOLM1 can be a valuable marker for the diagnosis of early HCC, especially for the diagnosis of AFP-negative HCC patients. In recent years, some studies have even shown that GOLM1 is superior to AFP in diagnosing early HCC [18,19]. Many studies have proved that GOLM1 has high sensitivity and specificity for the diagnosis of HCC. Although the thresholds taken by researchers are different, we can roughly observe a range: the sensitivity $(\sim 69-83 \%)$ and the specificity $(\sim 73-$ 
97\%) [19-22]. In one multicenter study, Serum GOLM1 and AFP were compared in 4217 human subjects. GOLM1 was used in the diagnosis of HCC with a sensitivity of $74.6 \%$ and a specificity of $97.4 \%$ [18]. In the year 2005, Marrero $e t$ al. detected serum GOLM1 of 352 patients and found that the sensitivity and specificity of GOLM1 for the diagnosis of HCC were 69 and 75\%, respectively, at the best cut-off point of 10 relative units [22]. Tian et al. demonstrated no significant differences in serum GOLM1 levels between the early and advanced HCC groups (110.4 vs $102.8 \mu \mathrm{g} / \mathrm{l}$ ) [20], indicating that high levels of GOLM1 could be detected in the serum of early HCC patients. These findings confirmed that GLOM1 had high sensitivity in the diagnosis of HCC. Zhao et al. demonstrated that fucosylated GOLM1 (Fuc-GOLM1) could be used as an excellent glycobiomarker for differential diagnosis of HCC and the combined detection of Fuc-GOLM1 and other biomarkers could improve the accuracy of diagnosis [23].

However, the clinical application of GOLM1 still faces some challenges for the early diagnosis of HCC. Liu et al. found that the serum expression levels of GOLM1 in HCC patients with cirrhosis were significantly higher than those in HCC patients without liver cirrhosis [24]. They suggested that it was possibly the cirrhotic background rather than the HCC itself that caused the increase of serum GOLM1 in HCC patients. One meta-analysis from Yang et al. including 11 studies showed that the sensitivity for serum GOLM1 in diagnosing HCC was 77\% (95\% CI: 75-79\%) and the specificity was 91\% (95\% CI: 90-92\%) [25]. This meta-analysis demonstrated that GOLM1 could be used as an independent diagnostic marker for HCC. However, significant heterogeneity was found in these 11 studies: five of the studies indicated that GOLM1 was superior to AFP as a diagnostic marker, while the other six studies had opposite or ambiguous findings. Therefore, it is necessary to conduct further research with rigorous design, large sample size and multi-regional cooperation to assess whether GOLM1 is the reliable marker for early diagnosis of HCC.

\section{Role of GOLM1 in the progression of HCC}

Sun et al. used immunohistochemistry to find that the expression of GOLM1 in HCC was closely related to tumor size, vascular invasion and tumor differentiation, suggesting that GOLM1 may enhance tumor invasion and metastasis to promote the progression of HCC [26]. Chen et al. demonstrated that GOLM1 could promote the proliferation and migration of HCC cell lines and promote the growth of mouse xenograft tumors [27]. Their study further indicated that the expression of GOLM1 could be regulated by mTORC1. mTOR inhibitors which block the upregulation of GOLM1 may be developed to treat HCC. Epithelial-mesenchymal transition (EMT) refers to the process of transforming epithelial cells into mesenchymal cells, which frequently occurs in the initiation of tumor invasion and metastasis [28]. E-cadherin and vimentin are two molecular markers related to EMT. Bao et al. found a negative correlation between GOLM1 and E-cadherin expression and a positive correlation with vimentin expression in HCC [29], suggesting that GOLM1 promotes EMT formation and is associated with aggression of HCC. Yang et al. examined the expression levels of GOLM1 protein and mRNA in cell lines with different metastatic potentials to explore the relationship between GOLM1 and tumor metastasis [30]. They found higher GOLM1 expression level in the more aggressive cells MHCC97H and HCCLM3, while lower GOLM1 level in the less aggressive cell Bel-7404 and the noninvasive cell L-O2. Ye et al. found that GOLM1 was involved in mediating EGFR/RTK signaling (Figure 2) [31]. After ligand binding to activate EGFR, endocytic EGFR bound to cytoplasm-dispersed GOLM1 to form endosomes, which promoted the return of EGFR to the cell membrane and reduced the degradation of EGFR in lysosomes. In addition, GOLM1 also increased the expression of MMP9 and inhibited E-cadherin levels, which were the two pivotal downstream effectors of RTK signaling pathways in tumor invasion and metastasis. It is suggested that overexpression of GOLM1 inhibits the normal metabolism of EGFR/RTK in cells, increasing their expression on the membrane and prolonging the activation of downstream signaling pathways to promote the growth, invasion and metastasis of HCC.

Several other studies have shown when the expression of GOLM1 is inhibited, HCC cell migration and invasion were suppressed significantly [32,33]. Matrix metalloproteinases have proteolytic activity to degrade the extracellular matrix and basement membrane and promote tumor invasion and metastasis. MMP-13 is one member of this family that is highly expressed in malignant tumors such as breast cancer and esophageal cancer to promote tumor invasion and distant metastasis [34,35]. It was reported that GOLM1 promoted cell invasion through the upregulation of MMP-13 and GOLM1 enhanced MMP-13 expression through CREB-mediated transcription activation [33], which indicated that the abnormal expression of GOLM1-CREB-MMP13 signaling pathway enhanced HCC cell infiltration. Zhang et al. showed that GOLM1 could be directly targeted by miR-382 [36]. miR-382 mediated downregulation of GOLM1 might inhibit migration and invasion of HCC cells. Zhao et al. 


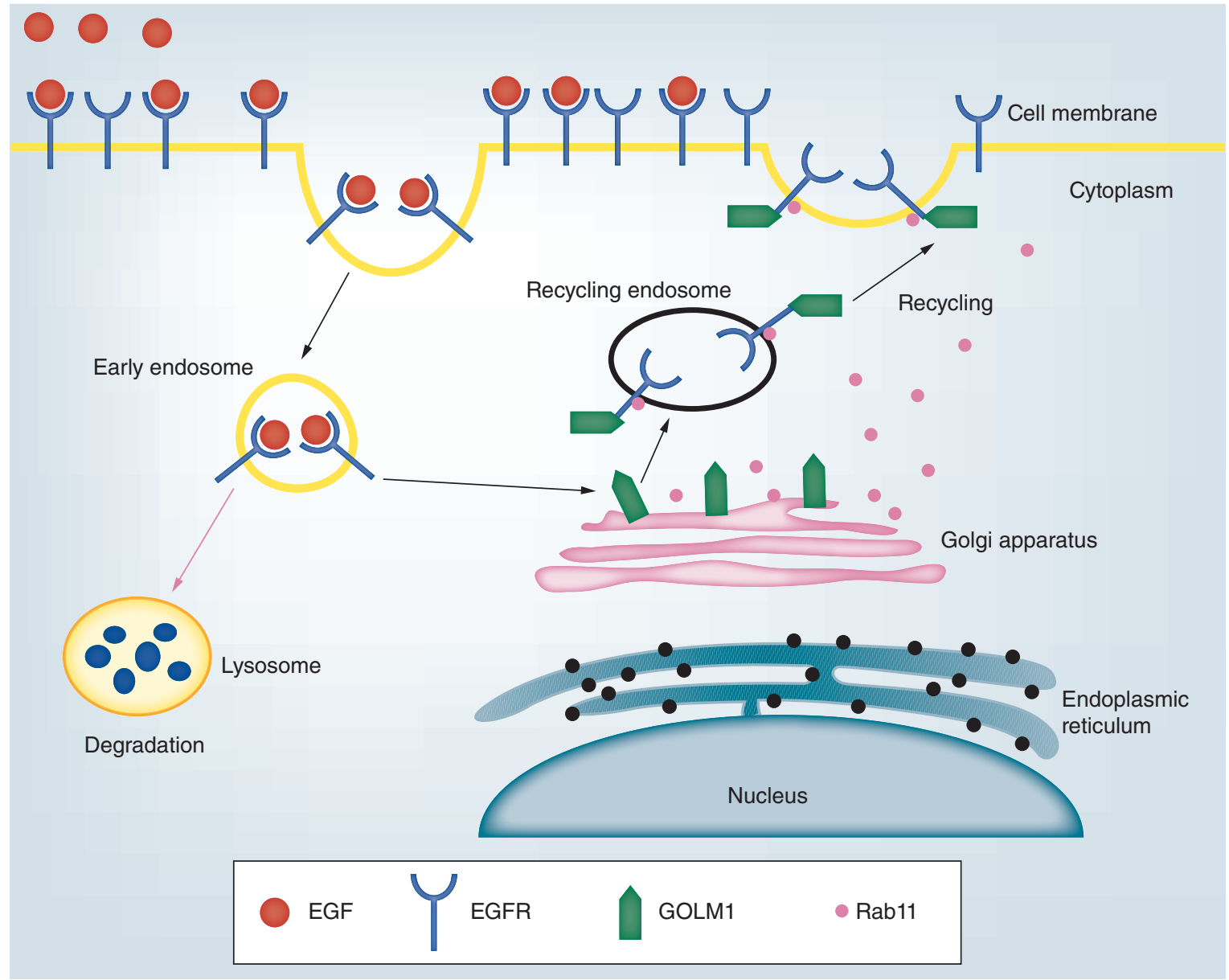

Figure 2. A brief illustration of GOLM1-mediated EGFR recycling. Following EGF binding, cell surface receptor EGFR is destined for internalization for receptor recycling or lysosome-mediated degradation. GOLM1 is localized in the Golgi apparatus and GOLM1-mediated recycling of EGFR is dependent on Rab11.

demonstrated that miR-493-5p could inhibit the proliferation of HCC cells, partly through directly inhibiting GOLM1 mRNA and protein levels [37]. Yang et al. found significant changes in the morphological phenotypes and related markers after the inhibition of GOLM1 [30]. Specifically, GOLM1 reduced the expression of the two key EMT factors N-cadherin and E-cadherin to decrease cell adhesion forces and to promote cancer cell motility. These findings suggested that GOLM1 silencing represents a novel therapy method to suppress proliferation and metastasis of tumors [32]. Zhou et al. provided the first experimental evidence to demonstrate that GOLM1 might be an important factor in the inhibitory regulation of autophagy, which indicated that GOLM1 was involved in the progression of GOLM1-related hepatoma [38]. Zhang et al. used stealth RNAi to target GOLM1 gene sequences to significantly reduce the expression of GOLM1 [39]. They found that knockdown of GOLM1 in HepG2 cells and Bel7402 cells could inhibit cell proliferation and induce apoptosis.

We conclude that the increase of GOLM1 expression may be closely related to tumor size, differentiation and vascular invasion, suggesting that GOLM1 may play an important role in the progression and metastasis of HCC.

\section{Significance of GOLM1 in the prognosis of HCC}

Prognostic biomarkers are used to predict the possibility of recurrence or progression in cancer patients. The identification of prognostic biomarkers in cancers could potentially lead to prognostic predictions for patients. This may be helpful to realize personalized medicine for cancer. So far, effective prognostic biomarkers for HCC are still lacking $[40,41]$.

It has been reported that increased expression of GOLM1 is closely associated with malignant biological characteristics and is an independent prognostic factors for poor overall survival [26,29,42]. Some studies demonstrate 
that GOLM1 is also of great value in predicting postoperative prognosis in patients with $\mathrm{HCC}$ [43,44]. It was reported that GOLM1 was maintained at a certain level 1 week after surgery in HCC patients, but decreased significantly 1.5-2 years after surgery, to the level lower than that in the liver cancer group and slightly higher than the normal group [45]. Some other studies also confirm that GOLM1 shows a downward trend after surgery or after treatment in HCC patients. GOLM1 level increases again when cancer recurs. Higher postoperative serum GOLM1 levels are significantly associated with an increased 5-year recurrence rate $[18,44,45]$.

It is difficult to predict the outcome of transcatheter arterial chemoembolization (TACE) in patients with negative AFP or low AFP concentrations. Ai et al. demonstrated that the serum GOLM1 levels in patients with a rapid disease progression were significantly higher than those in patients who achieved remission 30 days following TACE treatment [46]. The dynamic variations of serum GOLM1 levels may accurately represent the patient's condition, suggesting that it may be used in clinical testing for its important clinical application value in predicting the therapeutic effect of TACE in patients with AFP-negative or AFP levels $<400 \mu \mathrm{g} / \mathrm{l}$. Ye et al. also demonstrated that increased expression of GOLM1 was associated with multinodular tumors and microvascular invasion and decreased overall survival and time to tumor recurrence in patients [31]. These findings demonstrate that GOLM1 can be used as an independent prognostic risk factor and that higher levels suggests a poor prognosis. However, other studies show no significant correlation between GOLM1 expression and patients's survival. Sun et al. showed that GOLM1 expression level was closely associated with aggressive characteristics of HCC [26], but had no significant correlation with the overall survival in 36 HCC patients. Riener et al. reported that stronger GOLM1 expression significantly correlated with a higher tumor grade, but without significant correlation with survival or other clinicalpathological parameters in 170 HCC patients [43]. The small sample size and a lack of uniform follow-up may be responsible for the differences in the association of GOLM1 expression with tumor behavior and prognosis among these studies $[26,43]$.

There is limited information on the efficacy of surveillance strategies for predicting prognostic outcomes in resection HCC patients and it is mainly recommended for high-risk patients [47]. AFP has been widely used for predicting post-hepatectomy outcomes in HCC patients. However, Shim et al. showed that preoperative AFP levels had no significant correlation with survival [48]. Liang et al. believed that miR-27b and GOLM1 might be more conclusive than AFP as a prognostic marker [49]. We suggest that GOLM1 may complement current monitoring methods to predict outcomes of HCC patients after resection. Further studies including large samples and uniform follow-up are needed to verify the prognostic significance of GOLM1 expression in HCC.

\section{Role of GOLM1 in the immune system}

The immunological responses have been proved to play an important role in tumor development, growth and treatment. Immunotherapy as a new treatment option for HCC has attracted increasing attention. The following reports demonstrate the role of GOLM1 in the immune response.

Th1 cells are thought to mediate antitumor immunity. They mainly enhance the initiation and expansion of $\mathrm{CD}^{+} \mathrm{T}$ cells by secreting IFN- $\gamma$ and chemokines and recruit natural killer cells and Type I macrophages to tumor sites to eradicate tumors [50-54]. Tang et al. found that GOLM1 may inhibit the tumor suppressor effect of Th1 cells in cancer tissues by downregulating the expression of IL-12A [55], which might provide a new target for the prevention and treatment of tumor. Kim et al. speculated that the sGOLM1 in HCC may have a pathological effect in inhibiting IL-12 production by dendritic cells to prevent $T$ cells from responding to malignant development [56]. Hepatitis $\mathrm{C}$ virus (HCV) infection is one of the leading causes of HCC. Zhang et al. reported that GOLM1 could repress host innate immune response to promote $\mathrm{HCV}$ replication by facilitating mitochondrial antiviral signaling protein and TNF receptor-associated factor 6 degradation [57], which suggests that GOLM1 may be a novel potential antiviral target for the prevention and treatment of HCV-related diseases. Many studies have demonstrated that blocking of STAT3 activation could downregulate PD-L1 expression [58-60]. We previously found that knockdown of GOLM1 could downregulate the phosphorylation level of STAT3 [YAN J ET AL., UNPUBLISHED DATA]. Therefore, we speculate that it is possible to regulate the expression of PD-L1 through the modulation of GOLM1. We plan to conduct further research and verify whether the GOLM1-STAT3-PD-L1 axis exists. If it is confirmed to be true, it could provide a new intervention target for HCC immunotherapy.

These studies indicate that GOLM1 is closely related to the immune response. Further exploration of the role of GOLM1 in the immune response will help to understand its function and to identify novel targets for HCC immunotherapy. 


\section{Role of GOLM1 in the treatment of HCC}

Oncolytic adenoviruses have recently shown promising prospects for treating human cancers. However, traditional oncolytic adenoviruses lack the targeting ability for HCC. Wang et al. successfully constructed a novel GOLM1regulated oncolytic adenovirus and demonstrated that the GOLM1-regulated oncolytic adenovirus had higher infectivity for HCC cells than common oncolytic adenovirus [61]. It also exerted a more efficient growth-suppressing effect on HCC cells both in vitro and in vivo than common oncolytic virus. Cancer stem cells are very important for the development, maintenance and recurrence of cancer. Zhang et al. showed that GOLM1-regulated oncolytic adenovirus caused greater cytotoxicity and stronger oncolytic effect in liver cancer stem cell-like cells compared with common oncolytic viruses [62]. Therefore, the novel GOLM1-regulated oncolytic virus may be a promising anticancer agent candidate for future therapy of HCC.

\section{Conclusion}

The role of GOLM1 in the diagnosis, progression and prognosis assessment of HCC and its impact on the body's immune response have received growing attention. Many important findings have been achieved from relevant studies in these areas. In addition, there are also some reports on the role of GOLM1 in the treatment of HCC. GOLM1 may become a new intervention target for therapy of HCC. In summary, given its strong association with HCC, GOLM1 is an important molecule that deserves further research.

\section{Future perspective}

GOLM1 has attracted great attention since its discovery, but there are still many problems to be solved before GOLM1 can be accepted in clinical practice. The first issue is to understand the specific biological characteristics, secretion mechanisms and functions of GOLM1. At present, there is limited knowledge about the biological and clinical characteristics of GOLM1. Another key urgent issue is to conduct a multi-center and large-scale clinical study to evaluate the clinical value of GOLM1 in HCC.

\section{Executive summary}

- Hepatocellular carcinoma (HCC) is one of the most common causes of cancer-related deaths worldwide. Most HCC patients are diagnosed at the advanced stage which leads to the poor prognosis. It is necessary to explore effective methods of early diagnosis and identify valuable evaluation indicators.

- GOLM1 is one of the most promising biomarkers for early diagnosis and for the prediction of prognosis of HCC.

- GOLM1 is expressed in most tissues, it may belong to the housekeeping genes. However, its expression level is significantly different in various tissues, indicating that GOLM1 may have effective regulatory abilities and tissue-specific functions.

- The expression level of GOLM1 is significantly increased in HCC, indicating that it may be a valuable marker for early diagnosis of HCC, especially for the diagnosis of alpha-fetoprotein-negative HCC patients.

- The higher expression level of GOLM1 is related to larger tumor size, vascular invasion and tumor differentiation in HCC, suggesting that GOLM1 may enhance tumor invasion and metastasis to promote the progression of HCC.

- GOLM1 is closely associated with malignant biological characteristics and is an independent prognostic factor for poor overall survival in HCC.

- Immunotherapy as a potential new treatment option for HCC has attracted growing attention. GOLM1 is closely related to the immune response, thus prompting further exploration of the role of GOLM1 in the immune system.

- Given its strong association with HCC, GOLM1 is an important molecule that deserves further research.

\footnotetext{
Author contributions

J Yan and B Zhou consulted the literature, analyzed the data, summarized and wrote the manuscript. H Li and L Guo analyzed the literature data and revised the manuscript. Q Ye supervised the design of this review, analyzed the literature data, made a summary and revised the manuscript. All authors approved the final version of the manuscript.
}

Financial \& competing interests disclosure

This work was supported in part by the Program of Shanghai Subject Chief Scientist (no. 16XD1400800) and the National Natural Science Foundation of China (no. 81572301, 81502487, 81802893 and 81871924). The authors have no other relevant affiliations or financial involvement with any organization or entity with a financial interest in or financial conflict with the subject matter or materials discussed in the manuscript apart from those disclosed.

No writing assistance was utilized in the production of this manuscript. 


\section{Open access}

This work is licensed under the Attribution-NonCommercial-NoDerivatives 4.0 Unported License. To view a copy of this license, visit http://creativecommons.org/licenses/by-nc-nd/4.0/

\section{References}

Papers of special note have been highlighted as: • of interest; $\bullet \bullet$ of considerable interest

1. Forner A, Reig M, Bruix J. Hepatocellular carcinoma. Lancet 391(10127), 1301-1314 (2018).

2. Li H, Zhang P, Luo J et al. Chondroitin sulfate-linked prodrug nanoparticles target the Golgi apparatus for cancer metastasis treatment. ACS Nano 13(8), 9386-9396 (2019).

3. Yu RY, Xing L, Cui PF et al. Regulating the Golgi apparatus by co-delivery of a COX-2 inhibitor and brefeldin A for suppression of tumor metastasis. Biomater. Sci. 6(8), 2144-2155 (2018).

4. Sai WL, Yao M, Shen SJ et al. Dynamic expression of hepatic GP73 mRNA and protein and circulating GP73 during hepatocytes malignant transformation. Hepatobiliary Pancreat. Dis. Int. doi:10.1016/j.hbpd.2020.02.009 (2020) (Epub ahead of print).

5. Liu Y, Zhou S, Shi J et al. c-Myc transactivates GP73 and promotes metastasis of hepatocellular carcinoma cells through GP73-mediated MMP-7 trafficking in a mildly hypoxic microenvironment. Oncogenesis 8(10), 58 (2019).

6. Gai X, Tang B, Liu F et al. mTOR/miR-145-regulated exosomal GOLM1 promotes hepatocellular carcinoma through augmented GSK-3beta/MMPs. J. Genet. Genomics 46(5), 235-245 (2019).

7. Ke MY, Wu XN, Zhang Y, Wang S, Lv Y, Dong J. Serum GP73 predicts posthepatectomy outcomes in patients with hepatocellular carcinoma. J. Transl. Med. 17(1), 140 (2019).

8. Kladney RD, Bulla GA, Guo L et al. GP73, a novel Golgi-localized protein upregulated by viral infection. Gene 249(1-2), 53-65 (2000).

- First report of the isolation and characterization of GOLM1.

9. Zhou Y, Li L, Hu L, Peng T. Golgi phosphoprotein 2 (GOLPH2/GP73/GOLM1) interacts with secretory clusterin. Mol. Biol. Rep. 38(3), 1457-1462 (2011).

10. Xia Y, Zhang Y, Shen M, Xu H, Li Z, He N. Golgi protein 73 and its diagnostic value in liver diseases. Cell Prolif. 52(2), e12538 (2019).

11. Ba MC, Long H, Tang YQ, Cui SZ. GP73 expression and its significance in the diagnosis of hepatocellular carcinoma: a review. Int. J. Clin. Exp. Pathol. 5(9), 874-881 (2012).

12. Iftikhar R, Kladney RD, Havlioglu N et al. Disease- and cell-specific expression of GP73 in human liver disease. Am. J. Gastroenterol. 99(6), 1087-1095 (2004).

13. Kladney RD, Tollefson AE, Wold WS, Fimmel CJ. Upregulation of the Golgi protein GP73 by adenovirus infection requires the E1A CtBP interaction domain. Virology 301(2), 236-246 (2002).

14. Puri S, Bachert C, Fimmel CJ, Linstedt AD. Cycling of early Golgi proteins via the cell surface and endosomes upon lumenal $\mathrm{pH}$ disruption. Traffic 3(9), 641-653 (2002).

15. Bachert C, Fimmel C, Linstedt AD. Endosomal trafficking and proprotein convertase cleavage of cis Golgi protein GP73 produces marker for hepatocellular carcinoma. Traffic 8(10), 1415-1423 (2007).

16. Wright LM, Yong S, Picken MM, Rockey D, Fimmel CJ. Decreased survival and hepato-renal pathology in mice with C-terminally truncated GP73 (GOLPH2). Int. J. Clin. Exp. Pathol. 2(1), 34-47 (2009).

17. Shi Y, Chen J, Li L et al. A study of diagnostic value of golgi protein GP73 and its genetic assay in primary hepatic carcinoma. Technol. Cancer Res. Treat. 10(3), 287-294 (2011).

18. Mao Y, Yang H, Xu H et al. Golgi protein 73 (GOLPH2) is a valuable serum marker for hepatocellular carcinoma. Gut 59(12), 1687-1693 (2010).

-• Demonstrates that GOLM1 is an accurate serum marker for the detection of hepatocellular carcinoma and its recurrence after surgery.

19. Zhou Y, Yin X, Ying J, Zhang B. Golgi protein 73 versus alpha-fetoprotein as a biomarker for hepatocellular carcinoma: a diagnostic meta-analysis. BMC Cancer 12, 17 (2012).

20. Tian L, Wang Y, Xu D et al. Serological AFP/Golgi protein 73 could be a new diagnostic parameter of hepatic diseases. Int. J. Cancer 129(8), 1923-1931 (2011).

21. Hu JS, Wu DW, Liang S, Miao XY. GP73, a resident Golgi glycoprotein, is sensibility and specificity for hepatocellular carcinoma of diagnosis in a hepatitis B-endemic Asian population. Med. Oncol. 27(2), 339-345 (2010).

22. Marrero JA, Romano PR, Nikolaeva $\mathrm{O}$ et al. GP73, a resident Golgi glycoprotein, is a novel serum marker for hepatocellular carcinoma. J. Hepatol. 43(6), 1007-1012 (2005).

23. Zhao Y, Zhang L, Huo L et al. Clinical significance of fucosylated GP73 in the differential diagnosis of hepatocellular carcinoma. Int. J. Biol. Markers 33(4), 439-446 (2018). 
24. Liu T, Yao M, Liu S et al. Serum Golgi protein 73 is not a suitable diagnostic marker for hepatocellular carcinoma. Oncotarget 8(10), 16498-16506 (2017).

25. Yang J, Li J, Dai W et al. Golgi protein 73 as a biomarker for hepatocellular carcinoma: a diagnostic meta-analysis. Exp. Ther. Med. 9(4), 1413-1420 (2015).

26. Sun Y, Yang H, Mao Y et al. Increased Golgi protein 73 expression in hepatocellular carcinoma tissue correlates with tumor aggression but not survival. J. Gastroenterol. Hepatol. 26(7), 1207-1212 (2011).

27. Chen X, Wang Y, Tao J et al. mTORC1 up-regulates GP73 to promote proliferation and migration of hepatocellular carcinoma cells and growth of xenograft tumors in mice. Gastroenterology 149(3), 741-752 e714 (2015).

28. Dong $\mathrm{Y}$, Zheng Q, Wang $\mathrm{Z}$ et al. Higher matrix stiffness as an independent initiator triggers epithelial-mesenchymal transition and facilitates HCC metastasis. J. Hematol. Oncol. 12(1), 112 (2019).

29. Bao YX, Cao Q, Yang Y et al. Expression and prognostic significance of golgiglycoprotein73 (GP73) with epithelial-mesenchymal transition (EMT) related molecules in hepatocellular carcinoma (HCC). Diagn. Pathol. 8, 197 (2013).

30. Yang Y, Liu Q, Zhang $\mathrm{H}$ et al. Silencing of GP73 inhibits invasion and metastasis via suppression of epithelial-mesenchymal transition in hepatocellular carcinoma. Oncol. Rep. 37(2), 1182-1188 (2017).

31. Ye QH, Zhu WW, Zhang JB et al. GOLM1 modulates EGFR/RTK cell-surface recycling to drive hepatocellular carcinoma metastasis. Cancer Cell 30(3), 444-458 (2016).

-. Reveals the functional role of GOLM1 in EGFR recycling and metastatic progression of hepatocellular carcinoma.

32. Liu Y, Zhang X, Sun T et al. Knockdown of Golgi phosphoprotein 2 inhibits hepatocellular carcinoma cell proliferation and motility. Oncotarget 7(16), 21404-21415 (2016).

33. Jin D, Tao J, Li D et al. Golgi protein 73 activation of MMP-13 promotes hepatocellular carcinoma cell invasion. Oncotarget 6(32), 33523-33533 (2015).

34. Shah M, Huang D, Blick T et al. An MMP13-selective inhibitor delays primary tumor growth and the onset of tumor-associated osteolytic lesions in experimental models of breast cancer. PLoS ONE 7(1), e29615 (2012).

35. Osako Y, Seki N, Kita Y et al. Regulation of MMP13 by antitumor microRNA-375 markedly inhibits cancer cell migration and invasion in esophageal squamous cell carcinoma. Int. J. Oncol. 49(6), 2255-2264 (2016).

36. Zhang S, Ge W, Zou G et al. MiR-382 targets GOLM1 to inhibit metastasis of hepatocellular carcinoma and its down-regulation predicts a poor survival. Am. J. Cancer Res. 8(1), 120-131 (2018).

37. Zhao J, Xu T, Wang F, Cai W, Chen L. miR-493-5p suppresses hepatocellular carcinoma cell proliferation through targeting GP73. Biomed. Pharmacother. 90, 744-751 (2017).

38. Zhou YY, Jiang JC, You J, Zhou LF. Effect of Golgi phosphoprotein 2 (GOLPH2/GP73) on autophagy in human hepatocellular carcinoma HepG2 cells. Tumour Biol. 36(5), 3399-3406 (2015).

39. Zhang YL, Zhang YC, Han W et al. Effect of GP73 silencing on proliferation and apoptosis in hepatocellular cancer. World J. Gastroenterol. 20(32), 11287-11296 (2014).

40. Zhao N, Guo M, Wang K, Zhang C, Liu X. Identification of pan-cancer prognostic biomarkers through integration of multi-omics data. Front. Bioeng. Biotechnol. 8, 268 (2020).

41. Daniele A, Divella R, Quaranta M et al. Clinical and prognostic role of circulating MMP-2 and its inhibitor TIMP-2 in HCC patients prior to and after trans-hepatic arterial chemo-embolization. Clin. Biochem. 47(3), 184-190 (2014).

42. Chen MH, Jan YH, Chang PM et al. Expression of GOLM1 correlates with prognosis in human hepatocellular carcinoma. Ann. Surg. Oncol. 20(Suppl. 3), S616-S624 (2013).

43. Riener MO, Stenner F, Liewen H et al. Golgi phosphoprotein 2 (GOLPH2) expression in liver tumors and its value as a serum marker in hepatocellular carcinomas. Hepatology 49(5), 1602-1609 (2009).

44. Hann HW, Wang M, Hafner J et al. Analysis of GP73 in patients with HCC as a function of anti-cancer treatment. Cancer Biomark. 7(6), 269-273 (2010).

45. Mao YL, Yang HY, Xu HF et al. [Significance of Golgi glycoprotein 73, a new tumor marker in diagnosis of hepatocellular carcinoma: a primary study]. Zhonghua Yi Xue Za Zhi 88(14), 948-951 (2008).

46. Ai N, Liu W, Li ZG, Ji H, Li B, Yang G. High expression of GP73 in primary hepatocellular carcinoma and its function in the assessment of transcatheter arterial chemoembolization. Oncol. Lett. 14(4), 3953-3958 (2017).

47. Ando E, Kuromatsu R, Tanaka M et al. Surveillance program for early detection of hepatocellular carcinoma in Japan: results of specialized department of liver disease. J. Clin. Gastroenterol. 40(10), 942-948 (2006).

48. Shim JH, Yoon DL, Han S et al. Is serum alpha-fetoprotein useful for predicting recurrence and mortality specific to hepatocellular carcinoma after hepatectomy? A test based on propensity scores and competing risks analysis. Ann. Surg. Oncol. 19(12), 3687-3696 (2012).

49. Liang $\mathrm{H}$, Ai-Jun J, Ji-Zong $\mathrm{Z}$ et al. Clinicopathological significance of miR-27b targeting Golgi protein 73 in patients with hepatocellular carcinoma. Anticancer Drugs 30(2), 186-194 (2019). 
50. Nishimura T, Iwakabe K, Sekimoto $\mathrm{M}$ et al. Distinct role of antigen-specific T helper Type 1 (Th1) and Th2 cells in tumor eradication in vivo. J. Exp. Med. 190(5), 617-627 (1999).

51. Jenner RG, Townsend MJ, Jackson I et al. The transcription factors T-bet and GATA-3 control alternative pathways of T-cell differentiation through a shared set of target genes. Proc. Natl Acad. Sci. USA 106(42), 17876-17881 (2009).

52. Corthay A, Skovseth DK, Lundin KU et al. Primary antitumor immune response mediated by CD4+ T cells. Immunity 22(3), 371-383 (2005).

53. Palucka K, Banchereau J. Cancer immunotherapy via dendritic cells. Nat. Rev. Cancer 12(4), 265-277 (2012).

54. Kim HJ, Cantor H. CD4 T-cell subsets and tumor immunity: the helpful and the not-so-helpful. Cancer Immunol. Res. 2(2), 91-98 (2014).

55. Tang QF, Ji Q, Tang Y et al. Golgi phosphoprotein 2 down-regulates the Th1 response in human gastric cancer cells by suppressing IL-12A. Asian Pac. J. Cancer Prev. 14(10), 5747-5751 (2013).

56. Kim HJ, Lv D, Zhang Y, Peng T, Ma X. Golgi phosphoprotein 2 in physiology and in diseases. Cell Biosci. 2(1), 31 (2012).

57. Zhang X, Zhu C, Wang T et al. GP73 represses host innate immune response to promote virus replication by facilitating MAVS and TRAF6 degradation. PLoS Pathog. 13(4), e1006321 (2017).

58. Zhang W, Zhang J, Zhang Z et al. Overexpression of indoleamine 2,3-dioxygenase 1 promotes epithelial-mesenchymal transition by activation of the IL-6/STAT3/PD-L1 pathway in bladder cancer. Transl. Oncol. 12(3), 485-492 (2019).

59. Wolfle SJ, Strebovsky J, Bartz H et al. PD-L1 expression on tolerogenic APCs is controlled by STAT-3. Eur. J. Immunol. 41(2), 413-424 (2011).

60. Song TL, Nairismagi ML, Laurensia Y et al. Oncogenic activation of the STAT3 pathway drives PD-L1 expression in natural killer/T-cell lymphoma. Blood 132(11), 1146-1158 (2018).

61. Wang Y, Liu T, Huang P et al. A novel Golgi protein (GOLPH2)-regulated oncolytic adenovirus exhibits potent antitumor efficacy in hepatocellular carcinoma. Oncotarget 6(15), 13564-13578 (2015).

62. Zhang X, Meng S, Zhang R et al. GP73-regulated oncolytic adenoviruses possess potent killing effect on human liver cancer stem-like cells. Oncotarget 7(20), 29346-29358 (2016). 
